\title{
A Multiwavelength Study of CC Eridani
}

\author{
O. B. Slee, ${ }^{1}$ E. Budding, ${ }^{2,3}$ B. D. Carter, ${ }^{4}$ M. W. Mengel, ${ }^{4}$ I. Waite, ${ }^{4}$ and J.-F. Donati ${ }^{5}$ \\ ${ }^{1}$ Australia Telescope National Facility, PO Box 76, Epping NSW 2121, Australia \\ (e-mail: Bruce.Slee@atnf.csiro.au) \\ ${ }^{2}$ Carter Observatory, P.O. Box 2909, Wellington, New Zealand \\ ${ }^{3}$ currently at Physics Department, 18 March University of Canakkale, Turkey \\ (e-mail: ebudding@comu.edu.tr) \\ ${ }^{4}$ Centre for Astronomy, Solar Radiation and Climate, Faculty of Sciences, University of Southern \\ Queensland, Toowoomba QLD 4350, Australia (e-mail: carterb@usq.edu.au, mengelm@usq.edu.au) \\ ${ }^{5}$ Observatoire Midi-Pyrénées, 14 avenue Edouard Belin, 31400 Toulouse, France \\ (e-mail: jean-francois.donati@ast.obs-mip.fr)
}

Received 2003 May 8, accepted 2003 October 13

\begin{abstract}
Radio and optical observations from December 2001 and January 2002 of the active RS CVnlike binary CC Eri are presented. The star was monitored at 4.80 and $8.64 \mathrm{GHz}$ over $3 \times 12 \mathrm{~h}$ allocations with the Australia Telescope Compact Array on 28 to 30 December 2001. The Anglo-Australian Telescope was used for simultaneous optical spectropolarimetry during a $0.5 \mathrm{~h}$ period on 30 December. Data from four nights of broadband photometry gathered around the same period are also included in this present multiwavelength study.

The low levels of radio emission were circularly polarised at $\sim 20 \%$ with slightly positive spectral indices of $\sim 0.26$. Two flare-like increases were observed on successive nights with steep positive spectral indices and no detectable polarisation. Cross-correlation analysis of the 4.80 and $8.64 \mathrm{GHz}$ intensities over the stronger flare showed that the higher frequency emission preceded that at the lower frequency by $\sim 5 \mathrm{~min}$, a result consistent with the propagation of a hydromagnetic disturbance outwards through the corona. On the same night, a significant cross-correlation in the 'quiescent' emission indicates the presence of micro-flaring, although its low intensity does not permit the evaluation of a time delay. The emission parameters on the three nights are compatible with a gyrosyncrotron mechanism, in which the radio source becomes optically thick during strong flaring. We develop a simple model, which is based on assuming that the number of radiating electrons is a given function of the magnetic field in the source region, and derive feasible values for the field, source radius, and number of emitting electrons, which are not strongly dependent on the field modelling function or the aspect ratio of the source. Spectropolarimetry demonstrates the presence of a strong surface magnetic field. Optical photometry, covering a sufficient amount of the orbit, indicates a maculation region of significant size ( $\sim 14^{\circ}$ radius).

The results help develop a three-dimensional picture of a large stellar magnetically active region and encourage more detailed follow-up multiwavelength studies of this and similar stars.
\end{abstract}

Keywords: stars: activity — stars: coronae — stars: individual: CC Eri — radio continuum: stars techniques: miscellaneous — techniques: Zeeman Doppler Imaging.

\section{Introduction}

At the end of 2001, a co-ordinated observational effort, in both optical and radio wavelength ranges, was made on the short period, relatively near $(\sim 12 \mathrm{pc}), \operatorname{cool}(\mathrm{K} 7 \mathrm{~V}+\mathrm{M} 4 \mathrm{~V})$, low mass $\left(\sim 0.7 \mathrm{M}_{\odot}\right)$, BY Dra-type binary CC Eri $(=\mathrm{HD}$ 16157, HIP $11964, m_{\mathrm{V}} \approx 8.8$ ) over its orbital period (1.561 days). The observations arose from considerations of coronal emission and magnetic activity in cool, rapidly rotating 'active' dwarf stars. Radio emission from such stars is generally understood to come from gyrosynchrotron acceleration of electrons in moderate magnetic fields $\left(10^{-3}-10^{-2} \mathrm{~T}\right)$ at very high (brightness) temperatures of more than $\sim 10^{9} \mathrm{~K}$. Although the radio data constrain likely magnetic field strengths, it is very helpful to review results from other wavelengths to confirm estimates (or otherwise). In the present study, Zeeman Doppler Imaging (ZDI), using the Anglo-Australian Telescope, showed the presence of stellar magnetic fields at the surface of the primary star. Further evidence comes from the maculation ('starspot') effect, clearly visible in contemporaneous photometry of CC Eri (borne out also by historic light curves).

The present work recalls similar recent studies of the relatively bright variable HR 1817 (Budding et al. 2002). Results then obtained, together with surrounding recent research, encourage study of other active cool stars. A preliminary detection of CC Eri at $2.75 \mathrm{mJy}$ had been obtained during the course of the earlier observations of HR 1817. 
It is desirable to build up the observational database, to allow the physics of rapidly rotating dwarfs to be more effectively discussed (for example, see Ayres et al. 2000). The radio emission from such stars has been found to correlate with their rotation rates (Slee \& Stewart 1989), and, in binaries, we can expect variations linked to the orbital period. A previous ATNF-based multiwavelength study of the active cool binary CF Tuc (Budding et al. 1999) showed radio variations that correlated with starspot darkening. CC Eri is a non-eclipsing pair in a fairly close orbit, whose geometry might be deducible from photometric effects (evident also in its HIPPARCOS photometry). This binary is somewhat closer in its physical characteristics to YY Gem (which has similar low mass, but shorter period) and V824 Arae (similar mass and period, but more of a 'classical' RS CVn system).

A relevant scenario developed from multiwavelength studies of AB Dor, relates to Mestel and Spruit's (1987) giant loop model (see also Cameron \& Robinson 1989; Watson 1999). Mestel and Spruit's model predicts the sizes of coronal structures for a range of stellar parameters and it is of interest to check how well CC Eri fits in with these ideas. Another area of recent theoretical interest concerns coronal saturation. James et al. (2000) put limits on the range of stars where we may expect this, and CC Eri (one of their stars) falls into this range. Strong CaII and $\mathrm{H}_{\alpha}$ emission lines are indeed observed for CC Eri. Their intensities may also raise the intriguing idea of intercomponent magnetic field reconnection. The effects of such theoretically possible violent events would show across the whole spectrum. Pointers come from the recent study of Osten et al. (2002) of CC Eri, as well as previous studies (Osten et al. 2000). They discussed the connection between radio and other forms of emission, including time-delay effects and certain highly circularly polarised L-band outbursts attributed to strong flares. Osten et al. (2002) derived relatively high coronal densities from their EUV data, which they have had difficulty in reconciling with their microwave data.

The previous CF Tuc study (Budding et al. 1999) also showed strong inter-correlation of the emission across the range from 2.8 to $8.4 \mathrm{GHz}$. X-band $(8.6 \mathrm{GHz})$ variations tend to lead to similar variations at C-band $(4.8 \mathrm{GHz})$, usually by some tens of minutes. This suggests outwardpropagating disturbances through the corona of the active star. Putting these various activity effects into one coherent understanding is a compelling challenge.

\section{Data and Analysis}

\subsection{Radio Observations}

CC Eri was observed with the Compact Array at 4.80 and $8.64 \mathrm{GHz}$ on the three successive nights of 28,29 , and 30 December 2001, with the two frequencies being recorded simultaneously for $12 \mathrm{~h}$ each night. The six 22-m dishes in the ATCA are accurately mounted on an east-west baseline. They can be arranged at appropriate separations to obtain specific angular resolutions at

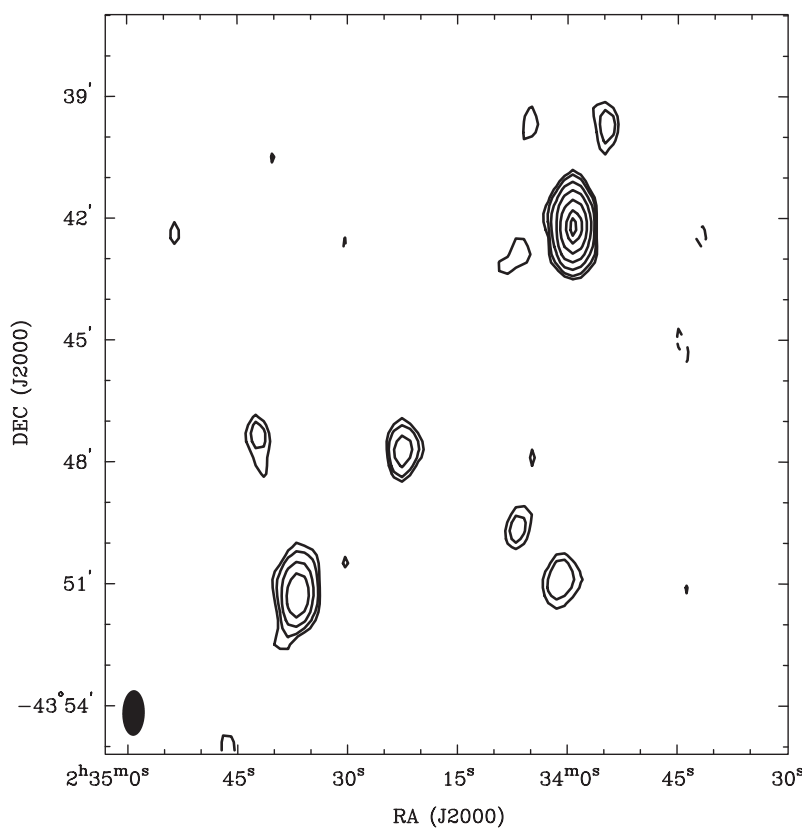

Figure 1 Cleaned radio map of the field surrounding CC Eri, using all the data for 29 December 2001. CC Eri lies towards the map centre on the line joining the two strongest field sources. The contour levels vary from 0.12 to $3.75 \mathrm{mJy} / \mathrm{beam}$, with the strong source to the NW having a peak brightness of $4.17 \mathrm{mJy} /$ beam. The RMS noise level over the blank area is $38.6 \mu \mathrm{Jy} / \mathrm{beam}$. The restoring beam, shown in the bottom-left corner, is $66.4 \times 34.9$ arcsec FWHM.

Table 1. Radio sources in the CC Eri field: Gaussian fit positions and flux densities

\begin{tabular}{|c|c|c|c|c|c|c|c|}
\hline \multirow[t]{3}{*}{ Source no. } & \multicolumn{7}{|c|}{ Position J2000 } \\
\hline & \multicolumn{3}{|c|}{ RA } & \multicolumn{3}{|c|}{ Dec } & \multirow{2}{*}{$\begin{array}{c}\text { Flux density (mJy) } \\
4.80 \mathrm{GHz}\end{array}$} \\
\hline & $\mathrm{h}$ & $\mathrm{m}$ & $\mathrm{s}$ & $\circ$ & 1 & " & \\
\hline 1 & 2 & 33 & 59.3 & -43 & 42 & 12 & 4.1 \\
\hline 2 & 2 & 34 & 22.7 & -43 & 47 & 45 & 0.7 \\
\hline 3 & 2 & 34 & 36.9 & -43 & 51 & 5 & 1.5 \\
\hline
\end{tabular}

Source No. 2 is CC Eri, 29 December 2000. Position errors (from the map in Fig. 1) are $\sim 0.5$ arcsec.

preassigned microwave observation frequencies (for further instrumental details see Manchester 1991). The total observation time of $36 \mathrm{~h}$ effectively covered the full binary period of 1.56 days. Five of the six antennae comprising the array were in a very compact configuration with a maximum spacing of $0.35 \mathrm{~km}$, resulting in shadowing on the two shortest baselines at low elevations. The much longer baselines with the sixth antenna were unaffected by shadowing.

A cleaned contour map of the field around CC Eri at $4.80 \mathrm{GHz}$ using the data for December 29 is shown in Figure 1 (also see Table 1). This map utilised only the data from the 10 baselines of the compact configuration, resulting in a restoring beam of $66.4^{\prime \prime} \times 34.9^{\prime \prime}$ with major axis in PA of $0.0 \mathrm{deg}$. There are 7 significant sources in this $14^{\prime} \times 14^{\prime}$ field with CC Eri lying close to the line joining the two strongest ones. 
An accurate position of CC Eri was obtained from 5 maps constructed from data from the 5 longest baselines (FWHM 1-2 arcsec). Five independent measurements were averaged to give a position:

$$
\begin{aligned}
\mathrm{RA} & =02: 34: 22.571 \pm 0.002 ; \\
\mathrm{Dec} & =-43: 47: 47.52 \pm 0.10
\end{aligned}
$$

The corrections for proper motion back to J2000.000 are $-0.011 \mathrm{~s}$ in RA and $+0.59^{\prime \prime}$ in Dec, resulting in a total difference of $0.063^{\prime \prime}$ between the radio and HIPPARCOS positions.

The radio data were reduced with the MIRIAD software (Sault \& Killeen 1996). First, cleaned, naturally weighted maps were made for each of the three nights; separate maps being produced from the data for the 10 compact spacings and the 5 long spacings. These maps established the presence of the star, its offset from the field centre and the existence of interfering field sources (see Fig. 1). The MIRIAD task UVFIT was then used to measure the flux densities at 4.80 and $8.64 \mathrm{GHz}$ for each of the 28-min integrations into which the night's observation was naturally broken.

It was immediately apparent that the sidelobes of the two strongest field sources were degrading the flux measurements at $4.80 \mathrm{GHz}$. This was effectively remedied by modelling the $4.80 \mathrm{GHz}$ field (with UVMODEL), using the model supplied by the clean components, but with a small mask over the position of CC Eri. These modelled data were then subtracted from the total data for the night, leaving substantially only the $u v$ components due to CC Eri itself. This was checked by mapping and cleaning the subtracted data set. This procedure was not needed at $8.64 \mathrm{GHz}$, because the two strong field sources were well outside the FWHM of the primary beam.

The final procedure was to measure the flux densities by applying UVFIT to the $u v$ data sets (subtracted at $4.80 \mathrm{GHz}$ ) for integrations of $28 \mathrm{~min}$ and $7 \mathrm{~min}$.

\subsection{Radio Light Curves}

Figure 2 shows the 28-min integrations at 4.80 and $8.64 \mathrm{GHz}$ over the three nights. The outstanding features of this plot are the high peaks near the ends of the second and, especially, the third nights. We interpret these as flares: this behaviour will be discussed in Section 3 . Table 2 shows that the average flux densities increased steadily over three days with the spectral index remaining mildly positive. We assume a power law of the form $S_{\nu} \sim \nu^{\alpha}$, where $\nu$ is the frequency and $\alpha$ the spectral index. The degree of polarisation was moderately high and righthanded (except on 29 December at $8.64 \mathrm{GHz}$ ). Such a high level of polarisation was also observed in this binary by Osten et al. (2002). The data are replotted as a function of orbital phase in Figure 3, using the ephemeris and period quoted in Strassmeier et al. (1993):

$$
\text { Min }=\text { HJD } 2430001.2905+1.56145 \mathrm{~d} \text { P. }
$$

Neglecting the two flares, there is no evidence of rotational modulation in this plot.
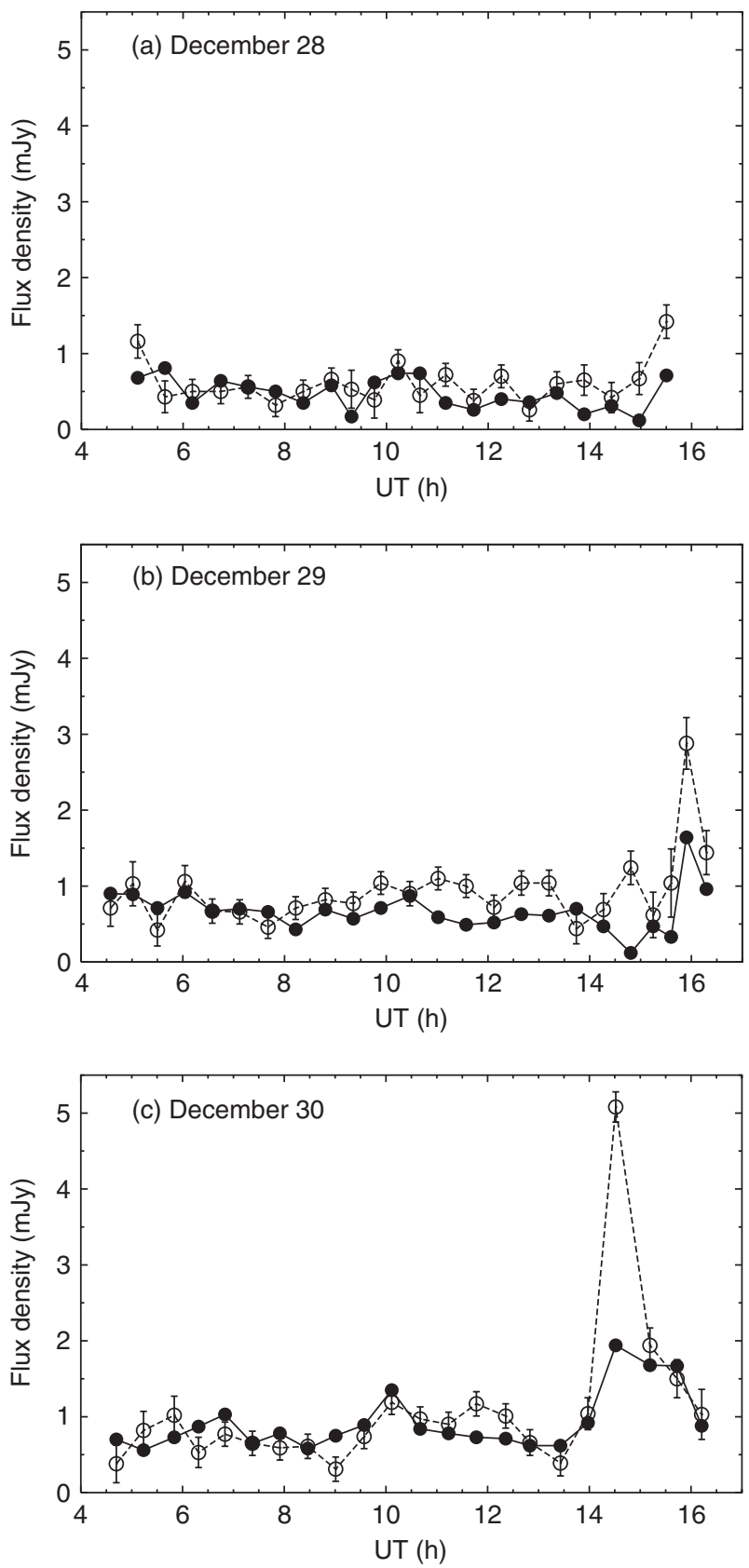

Figure 2 The flux densities from 28-min integrations. Filled circles denote $4.80 \mathrm{GHz}$ and open circles $8.64 \mathrm{GHz}$. Flares are evident towards the end of the observing intervals on December 29 and 30. To avoid confusion, error bars are added only to the $8.64 \mathrm{GHz}$ values. (They are similar for the $4.80 \mathrm{GHz}$ data.)

\subsection{Pronounced Radio Flares}

The occurrence of two radio flares has already been noted in Figure 2. The brighter flare of December 30 is shown in Figure 4 with the much improved time resolution provided by 7 -min integrations. The peak flux densities increase by factors of 4 and 8 above the average levels (Table 2) at 4.80 and $8.64 \mathrm{GHz}$, respectively; the total duration is approximately $22 \mathrm{~min}$. The maximum excess flux densities of $8.25 \mathrm{mJy}$ at $8.64 \mathrm{GHz}$ and $3.25 \mathrm{mJy}$ at $4.80 \mathrm{GHz}$ yield an optically thick spectrum with a spectral index of 
Table 2. Radio flux densities for each $12 \mathrm{~h}$ session

\begin{tabular}{lcccccrc}
\hline UT date & $\begin{array}{c}I_{4800} \\
(\mathrm{mJy})\end{array}$ & $\begin{array}{c}V_{4800} \\
(\mathrm{mJy})\end{array}$ & $\begin{array}{c}V_{4800} \\
(\%)\end{array}$ & $\begin{array}{c}I_{8640} \\
(\mathrm{mJy})\end{array}$ & $\begin{array}{c}V_{8640} \\
(\mathrm{mJy})\end{array}$ & $\begin{array}{r}V_{8640} \\
(\%)\end{array}$ & $\begin{array}{c}\text { Spectral } \\
\text { Index }\end{array}$ \\
\hline 28 Dec 01 & $0.50(0.035)$ & $0.16(0.038)$ & $32(9)$ & $0.58(0.038)$ & $0.19(0.038)$ & $33(9)$ & 0.25 \\
29 Dec 01 & $0.66(0.034)$ & $0.12(0.038)$ & $18(6)$ & $0.88(0.038)$ & $0.01(0.038)$ & $1(5)$ & 0.49 \\
30 Dec 01 & $0.90(0.034)$ & $0.27(0.039)$ & $30(5)$ & $1.02(0.039)$ & $0.18(0.039)$ & $18(5)$ & 0.21 \\
\hline
\end{tabular}

Bracketted values are the $1 \sigma$ uncertainties; $I$ and $V$ are the total intensity and circularly polarised intensities, respectively.

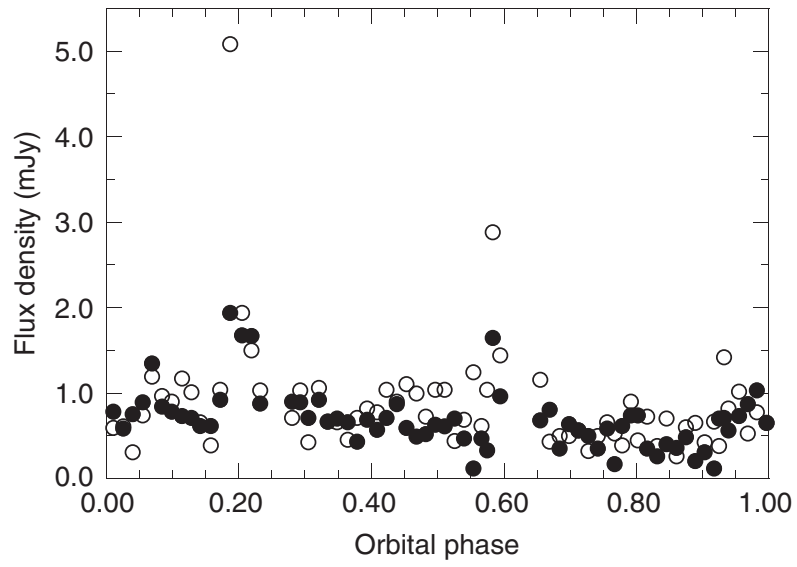

Figure 3 Flux densities for the three days are here plotted against orbital phase using the ephemeris given by Strassmeier et al. (1993). Filled circles denote $4.80 \mathrm{GHz}$ and open circles are $8.64 \mathrm{GHz}$. To avoid confusion, error bars have been omitted.

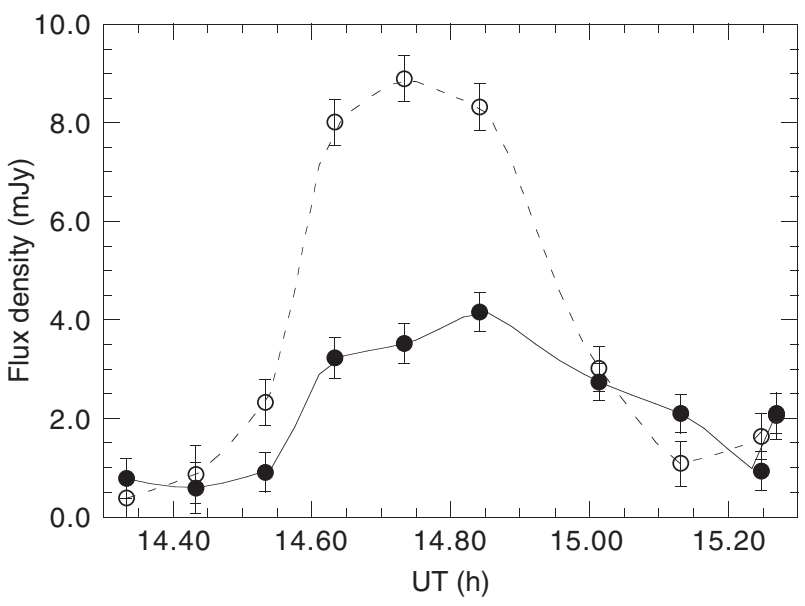

Figure 4 Flux densities of CC Eri over the flare interval on 30 December for integration times of $7 \mathrm{~min}$. The symbol representation is the same as for Figure 3.

$\alpha=1.6$. Figure 2 showed that similar spectral behaviour was exhibited by the weaker flare on December 29. The circularly polarised fraction at $8.64 \mathrm{GHz}$ at the flare peak was less than $5 \%$ ( $3 \sigma$ upper limit), which is consistent with optically thick gyrosynchrotron emission (Dulk 1985).

Using the above spectral index, the integrated power released between 1 and $8.64 \mathrm{GHz}$ can be put at $3.4 \times 10^{24} \mathrm{erg} \mathrm{s}^{-1}$. To make a useful estimate of the power emitted over the complete radio spectrum, one must postulate a spectral model. A reasonable model is that the spectral index remains at 1.6 up to a turnover at $20 \mathrm{GHz}$, whereupon the source becomes optically thin with a spectral index of -1.5 (see Fig. 2 of Dulk 1985). With this spectral model, the total power emitted over the frequency range $1-300 \mathrm{GHz}$ is $1.8 \times 10^{26} \mathrm{erg} \mathrm{s}^{-1}$. Using a half-power duration of the flare of $22 \mathrm{~min}$, the total radio energy emitted turns out to be $2.4 \times 10^{29} \mathrm{erg}$. This may be compared with strong, long-lasting flare emission from the Sun at these frequencies, which has a peak flux density of $\sim 10^{-18} \mathrm{~W} \mathrm{~m}^{-2} \mathrm{~Hz}^{-1}$ (Dulk 1985), corresponding to a radio power at the corona about three orders of magnitude below that from CC Eri. Assuming similar spectral models and flare durations, total emitted energies would differ by the same factor.

Another interesting feature of Figure 4 is the time shift between the centroids of the flare profiles at 4.80 and $8.64 \mathrm{GHz}$. The $4.80 \mathrm{GHz}$ profile appears to maximise about $7 \mathrm{~min}$ later. This is reminiscent of the behaviour seen in metre-wave Type II bursts from the Sun. As for the solar case, it is tempting to interpret this effect as due to the propagation of a shock wave outwards through the stellar corona. This would then be a more pronounced manifestation of the time delays in the coronae of active binaries and single stars noted by Budding et al. (2002).

\subsection{Correlation Analysis}

Our data for each day consisted of $22 \times 28$-min integrations, separated by 2-min observations of a secondary calibrator, the 4.80 and $8.64 \mathrm{GHz}$ data being recorded simultaneously. The typical error of a flux determination was $0.15 \mathrm{mJy}$ at both frequencies. Data from other active stars (Budding et al. 2002) have shown low amplitude variability at such wavelengths to be well correlated, even at times of apparent quiescence. They also showed significant time lags between the variations at the two frequencies.

Each of the three sets of data was examined for correlation between the 4.80 and $8.64 \mathrm{GHz}$ flux densities from the 28-min integrations, but excluding the flares near the ends of the data sets of December 29 and 30. Flux measurements are first interpolated onto a uniform time grid with spacing equal to the average time interval between the mid-times of the integrations. This is in order to minimise the effect of calibration breaks or other short recording irregularities. Central values of the correlation functions are derived by shifting the $4.80 \mathrm{GHz}$ set of interpolated flux 


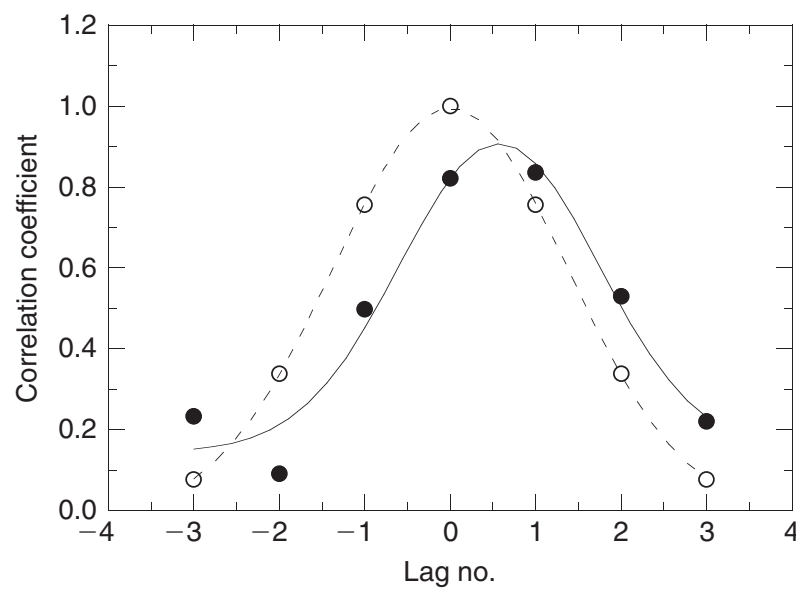

Figure 5 Correlation information derived from $\mathrm{C}$ and $\mathrm{X}$-band data over the strong flare on 30 December. The auto-correlation (open circles, dashed line) is a mean of the auto-correlations at both wavelengths. This and the cross-correlation (filled circles, continuous line) have been fitted with Gaussians to quantify their main characteristics. Note the relatively high peak of the cross-correlation and its shift with respect to a zero time-lag. The correlation values have been calculated at lag intervals of $7.985 \mathrm{~min}$.

densities, one step at a time, with respect to the $8.64 \mathrm{GHz}$ set. The process covers either direction about zero phase shift, computing the sum of products of corresponding ordinates at each step. The number of flux density points in the sets determines how far the shifts are reliably representative - with the 22 points examined here, we may consider a shift of \pm 3 steps about zero to be reasonably accurate against more general uncertainties. We found no significant correlation in the data sets of December 28 and 29 , but a correlation coefficient of 0.48 at zero lag was present in the stronger emission of December 30. However, the signal-to-noise levels of the correlations away from zero lag were too poor to define the cross-correlation function with enough accuracy to deduce a possible time shift between the intensity variations at the two frequencies. The correlation at zero lag does suggest the presence of microflaring.

We also computed correlations over the much shorter time interval encompassing the pronounced flare of December 30, the result being shown in Figure 5. Here we subdivided each 28-min integration in the 3 -h interval into four 7-min integrations and determined corresponding flux densities at the two frequencies. Each series was interpolated onto a uniform grid as before. Correlations have been evaluated to \pm 3 lags about the zero lag alignment, and results were fitted with Gaussians. The maximum cross-correlation coefficient of 0.90 occurs at a lag corresponding to $5 \pm 1 \mathrm{~min}$, with the $4.80 \mathrm{GHz}$ variations coming after those at $8.64 \mathrm{GHz}$. This result is naturally dominated by the flare itself and quantifies the shift, which can, in this case, be noticed by inspection of Figure 4 , where the X-band rise occurs shortly before that at C-band. Implications of these findings will be discussed in Section 4.
LS deconvolved Zeeman signature (4089 lines used)

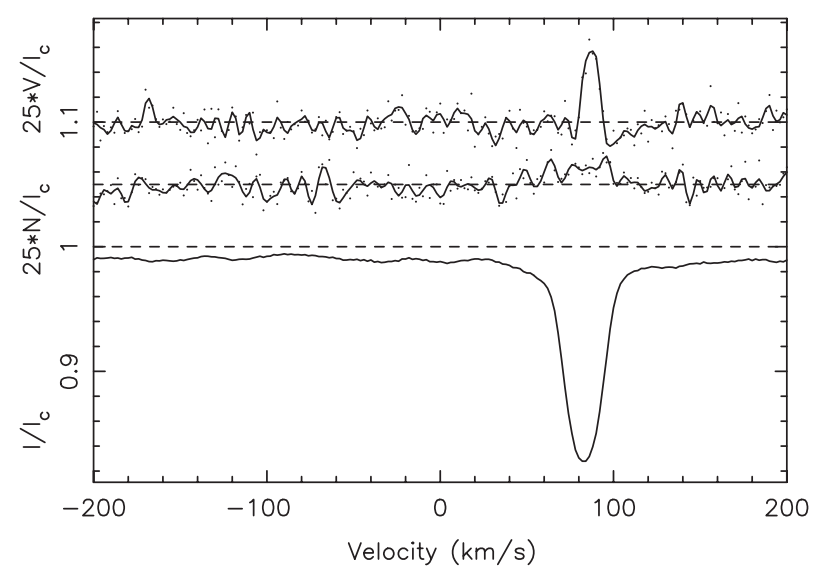

Figure 6 The optical polarisation and summed line intensity profile for CC Eri on 30 December 2001, obtained with the AngloAustralian Telescope, using Zeeman Doppler Imaging with least squares deconvolution.

\subsection{Optical Spectropolarimetry}

To investigate the presence of stellar magnetism associated with surface activity, optical spectropolarimetry of CC Eri was performed using the $3.9 \mathrm{~m}$ Anglo-Australian Telescope (AAT), equipped with the Semel polarimeter and an échelle spectrograph (Donati et al. 1997). The data were obtained as a sequence of four exposures on $30 \mathrm{Dec}$ 2001, from 10:11 to 10:28 UT.

Using the technique of Zeeman Doppler Imaging, with least squares deconvolution analysis (LSD) (Donati et al. 1997), the AAT data in Fig. 6 demonstrate the presence of a surface magnetic field contemporaneous with the radio observations. This observation is consistent with an earlier detection obtained for CC Eri in 1995 (Donati et al. 1997), and suggests that time-resolved optical spectropolarimetry could be employed to investigate in detail the star's magnetic field topology, as has been done for $\mathrm{K}$ dwarfs such as AB Dor (for example, Jardine, Cameron, \& Donati 2002).

The profiles in Figure 6 are plotted on a radial velocity scale, with the features of the primary star offset from zero due to CC Eri's orbital and space motions relative to the Earth. The upper profile is the composite Stokes V polarisation profile for approximately four thousand spectral lines in the available spectral range. This polarisation profile shows a strong feature (about ten times the local noise), indicating a magnetic field on the primary star. The middle plot further illustrates the relatively small noise level compared to the star's polarisation signature. The lowermost plot is the mean Stokes I line intensity profile. LSD combination of the $\mathrm{V}$ and I signals from about 4000 lines results in a significant improvement in the sensitivity of these measurements: more than a factor of $\sim 60$ compared to the use of a single line.

Note that the polarisation profile scale has been stretched relative to the intensity scale, for clarity. 


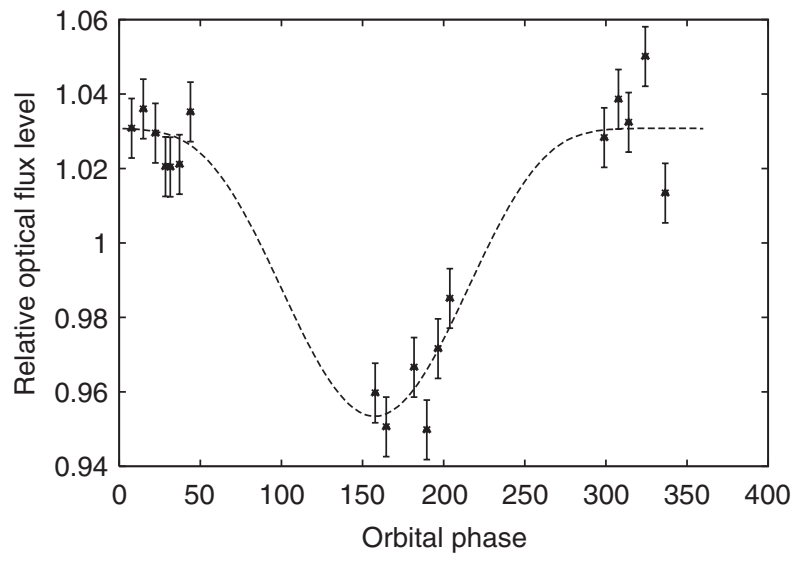

Figure 7 Broadband V light curve of CC Eri as obtained over the period 28 December 2001 to 2 January 2002. The observed points are shown with formal error bars corresponding to a $0.008 \mathrm{mag}$ standard deviation. Also shown is the best-fit circular spot model curve.

\subsection{V-band Photometry of CC Eri}

CC Eri was observed at the University of Southern Queensland's Mount Kent Observatory using a 40-cm Meade Schmidt-Cassegrain telescope and a Santa Barbara Instruments Group (SBIG) ST6 CCD camera (Waite 1999) equipped with a standard $\mathrm{V}$ filter. Observations were conducted over four nights between December 28 and January 2. The comparison and check stars used were HD16371 and SAO215945, respectively. The images were acquired using CCDOPS (from SBIG). The raw data were saved in SBIG's own file format. These were converted into FITS format using ToFits (Lane 1995) for reduction and analysis with IRAF.

Each image had the dark current removed (which included bias information) and was flat-fielded using a nightly master flat (usually 9 fields were combined using FLATCOMBINE). The stars in each frame were located by DAOFIND and the instrumental magnitudes determined using synthetic aperture photometry from DAOРнОт. The maximum good datum was set to $32 \mathrm{~K}$ - approximately half the full well capacity. If any data exceeded this value the image would be automatically rejected, in order to eliminate data that could be non-linear, to which ST6 cameras are prone when the well content exceeds half depth (Bessell 1996).

An aperture radius of 15 pixels was selected for the photometry of all stars, on the basis of their 'curve of growth' (Stetson 1990). This was checked, at random, throughout the analysis. The sky contribution to the signal was calculated using a 5 pixel wide circular annulus with an inner radius of 20 pixels. Resulting instrumental magnitudes were averaged in groups of three. Differential photometry determined the magnitudes of $\mathrm{CC}$ Eri at given points in time. The ephemeris used to find phases was that of Strassmeier et al. (1993) (see above). The resulting light curve is shown in Figure 7.

The mean V-band brightness of CC Eri was found to be $8.83 \mathrm{mag}$, with a variability of about $0.09 \mathrm{mag}$. The
Table 3. Photometric model for CC Eri

\begin{tabular}{|c|c|c|c|}
\hline \multicolumn{4}{|c|}{ Adopted (MS) binary representation } \\
\hline Parameter & & & Value \\
\hline Ref. V & & & 8.784 \\
\hline$L_{1}$ & & & 0.97 \\
\hline$L_{2}$ & & & 0.03 \\
\hline$r_{1}$ & & & 0.10 \\
\hline$r_{2}$ & & & 0.06 \\
\hline$i$ & & & 50.0 \\
\hline$u_{1}$ & & & 0.9 \\
\hline \multirow[t]{3}{*}{$M_{2} / M_{1}$} & & & 0.62 \\
\hline & \multicolumn{3}{|c|}{ Spot parameters } \\
\hline & Evans & Cutispoto & Present \\
\hline$\lambda$ & $148.6 \pm 1.1$ & $350.0 \pm 5$ & $157 \pm 5$ \\
\hline$\beta$ & $38 \pm 5$ & $57 \pm 23$ & 40 \\
\hline$\gamma$ & $21.1 \pm 0.2$ & $13.6 \pm 0.3$ & $13.5 \pm 0.7$ \\
\hline$\kappa$ & 0.0 & 0.0 & 0.0 \\
\hline
\end{tabular}

maximum brightness was observed at phase $\sim 0.95$ and the minimum at phase $\sim 0.47$.

\subsection{Photometric Analysis}

To interpret the light curve, a circular maculation formalism can be used (Budding 1977). These curve-fittings adopt a standard close binary (non-eclipsing) model, whose relevant parameters are listed in Table 3 using standard notation for reference visual magnitude, relative luminosities, radii relative to separation, axial inclination angle, limb darkening coefficient, and mass ratio (for example, see Budding 1993). We assume here a Main Sequence pair corresponding to the adopted spectral types (K7 and M4), and use a smooth interpolation of the empirical calibrations of Popper (1980).

Table 3 lists optimally fitted starspot model parameters for the present data, with their independent errors. The derived spot parameters, longitude $(\lambda)$, and angular radius $(\gamma)$, are shown in the lower part of Table 3 for an adopted latitude value $(\beta)$ consistent with that of Evans (1959).

The value of $\beta$ is a minimal size, since the fractional intensity of the spot's area is taken to be zero $(\kappa=0)$. We find the observed variations can be characterised by a dark spot of angular radius 13.6 deg located at longitude $157 \mathrm{deg}$ onward of the earth-facing meridian at phase zero and latitude $40 \mathrm{deg}$, in the sense of a right-handed frame located at the star's centre. The star's rotation axis is inclined at $50 \mathrm{deg}$ to the line of sight. (We cannot determine the orientation of this axis on the sky from photometric analysis.)

Our starspot model can also be used to derive parameters for CC Eri for the light curve of Cutispoto, Messina, and Rodono (2001), and that of Budding's (1977) fit to Evans' (1959) data. The data of Cutispoto et al. were taken in 1993, some 1896 rotations before the epoch of the present observations. Although we cannot be certain 
the spot feature observed by Cutispoto et al. is the same one observed by us, if this is indeed the case, Table 3 suggests that the spot has drifted and implies a differential rotation coefficient of about 0.004 , which may be compared with $\sim 0.005$ for the similar, but less tightly bound, pair II Peg (Henry et al. 1995).

\section{Discussion}

The relatively close distance of CC Eri means that the brightness temperature for $1 \mathrm{mJy}$ of radio flux would not be as high as for many comparable radio-emitting BY Dra variables. A key point in the calculation, of course, is the size of the emitting region: the smaller the adopted area, the greater the corresponding brightness temperature. The radio flux itself is associated with electrons energised to near relativistic speeds that then cool through a gyrosynchrotron process in moderate coronal magnetic fields. This 'quiescent' emission is undulatory over timescales of tens of minutes across the microwave spectrum, suggesting sizes not very large compared with those of the stellar systems involved. It appears consistent with energy propagated by magnetohydrodynamic waves traversing an active corona. Related X- to C-band time-shifts for the dwarf AB Dor, for example, are shorter than those obtained for classical RS CVn binaries. This points to the larger coronal structures that one might expect for subgiants and accords with calculated size-scales. We therefore associate observed flux levels with sources comparable to, or smaller than, stellar dimensions; perhaps on the same order, or somewhat larger than, the maculation regions accounting for the photometric variations. Using the Rayleigh-Jeans flux-brightness-temperature expression (Allen 1974)

$$
f_{v}=2 \frac{k v^{2} A}{c^{2} R^{2}} T_{\mathrm{b}},
$$

and, with cgs units and a source of area of $A \sim 3 \times 10^{20} \mathrm{~cm}^{2}$ at the distance of CC Eri ( $R=12 \mathrm{pc})$, an approximate value for $T_{\mathrm{b}}$, is $\sim 6 \times 10^{9} \mathrm{~K}$ at $4.8 \mathrm{GHz}$.

The approximations of Dulk (1985) can be usefully employed to sketch out more of this picture. First set Dulk's equation (36) into a logarithmic form. Taking a representative viewing angle as $\pi / 4$ and a power-law index $\delta=3$ for the electron energy spectrum, we can sum the absorption along a length $l$ down to the $\tau=1$ surface, to obtain

$$
20.65=\log l-4.24 \log v_{\mathrm{G}}+\log N_{\mathrm{R}}+3.24 \log B,
$$

where $v_{\mathrm{G}}$ is the frequency in $\mathrm{GHz}, B$ is the magnetic induction and $N_{\mathrm{R}}$ is the density of microwave radiating electrons (in cgs units).

These radiating electrons are usually related to a powerlaw energy distribution that is also observed due to X-ray emission effects (Gary 1985; Stewart et al. 1988; Drake, Simon, \& Linksy 1992; Dempsey et al. 1993; Franciosini \& Chiuderi Drago 1995). This power-law distribution can be tailored to the ambient thermal energy distribution at a representative point on the high energy tail of the latter, typically $10 \mathrm{keV}$ (Dulk 1985). This implies that the radiating electron population $N_{\mathrm{R}}$ is some fraction of the ambient plasma electron density $N_{\mathrm{e}}$, i.e.,

$$
N_{\mathrm{R}} / N_{\mathrm{e}}=\psi\left(E_{\mathrm{p}}, E_{\mathrm{mp}}\right),
$$

where we would expect to derive the fraction $\psi$ from relevant characteristics of the energy distribution, such as the peak energy of the ambient (thermal) plasma $E_{\mathrm{p}}$ and the matching point $E_{\mathrm{mp}}$. In practice, probably the most direct way to do this is to examine observed energy distributions and evaluate the relevant fraction by a suitable quadrature. As well as being direct, this allows the power-law index $\delta$ to be checked.

Dulk's (1985) equation (37) for the effective temperature can be used in our equation (1), since we are considering optical depth unity $\left(T_{\mathrm{b}}=0.632 T_{\mathrm{e}}\right)$, so that, for $1 \mathrm{mJy}$ of received flux, we find

$$
20.92=2.76 \log v_{\mathrm{G}}-0.76 \log B+2 \log L,
$$

where $L=l / \eta$ is a representative radius of the emitting area and $\eta$ is an 'aspect ratio'. In previous comparable studies (for example, Budding et al. 1999), the special case of $\eta=1$ was considered, but for a more compact circumstellar plasma it would be smaller than unity. We also add a coronal model constraint in the form

$$
\log N_{\mathrm{e}}=a \log B+b .
$$

Various values for $a$ and $b$ have been considered in earlier work (Slee et al. 1987; Vilhu et al. 1993; Budding et al. 1999). For the present we leave the values unfixed and consider their effects, noting however, from the cited studies, that $2 / 3 \leq a \leq 2$.

Let us write $\Lambda$ for $\log L, \Phi$ for $\log B$, take $4.8 \mathrm{GHz}$ as the reference frequency for Equation (4) and write $\Delta v$ for the transition to another frequency (e.g. 8.64 GHz). The corresponding log (flux) would be greater by $\alpha \Delta v$, using the standard power law form $f \sim\left(v / v_{0}\right)^{\alpha}$. From the (nonflaring) energy distribution of Osten et al. (2002) for EI Eri (which yields $\delta$ close to 3 ), we have measured a typical value of $\psi$ to be $10^{-2}$, while the same paper gives $12-14$ as the likely range for the derived value of $\log N_{\mathrm{e}}$. Osten et al. also showed emission measures for CC Eri in the range $10^{51}-10^{52} \mathrm{~cm}^{-3}$. Utilising the foregoing, and having such expected ranges of parameters in mind, we write

$$
\log N_{\mathrm{R}}=a \Phi+5.5
$$

as a suitable trial form to substitute into Equation (2). In order to relate this to the ambient plasma density, we introduce $\xi$ to allow for any changes to the trial constant. Note that $\xi$ also involves $\log \eta$ through Equation (2), as well as the ratio $\psi$ coming from Equation (3). Thus $\xi$ is increased by making the corona more dense and decreased by flattening out the aspect (i.e. decreasing $\eta$ ). In effect,

$$
\xi=b-5.5+\log \eta+\log \psi .
$$


We then find, from Equation (2),

$$
18.04=\Lambda+(3.24+a) \Phi-4.24 \Delta v+\xi .
$$

We can similarly re-express Equation (4) as

$$
19.04=2 \Lambda-0.76 \Phi+(2.76-\alpha) \Delta \nu .
$$

Equations (8) and (9) then yield:

$$
\Phi=\frac{17.04-2 \xi+(11.24-\alpha) \Delta v}{7.24+2 a} .
$$

This shows that at $4.8 \mathrm{GHz}(\Delta v=0)$, for the considered range of $a$, the magnetic field is relatively insensitive to the density or structure and likely to be generally less than 100 gauss; or slightly greater for fairly compact or low density structures. It will be greater for $8.64 \mathrm{GHz}(\Delta \nu=0.26)$, and this goes with the deeper coronal region from which the higher frequency radiation emerges. From Equation (9) we also observe

$$
\Lambda=9.52-(1.38-0.5 \alpha) \Delta v+0.38 \Phi,
$$

which indicates that size scales are of the order of several $\times 10^{10} \mathrm{~cm}$ in radius, relatively independently of the field (which itself does not vary enormously from $10^{2} \mathrm{G}$ in this scenario for microwave emission), and are smaller at higher frequencies, with a weak dependence on the (low) spectral index. These sizes, that are of order $10^{-1}$ of the stellar radius, would be made smaller, at a given frequency, by increasing the base density or elongating the structure [i.e. decreasing the required $\beta$ by increasing $\xi$ in Equation (10)].

The results from Equations (10), (11) and (5) (with $\log \psi=-2$, i.e. $b=7.5)$, using different plausible values for $a$ and $\xi$, are summarised in Table 4, where we have taken $\alpha=0.32$ as the average of the values listed in Table 2 . The values listed for $N_{\mathrm{e}}$ will ensure that Razin-Tsytovich suppression will not be relevant above a frequency of $1 \mathrm{GHz}$, at least for $a \leq 1$.

The ambient densities of Table 4 show an increase in the region of higher frequency emission, occupying a smaller volume further down in the corona. These densities are generally less than those found by Osten et al. (2002) for the EUV emitting region by up to three orders of magnitude. On the other hand, if we use the length scales of Table 4 to form an estimate of the volumes, then the corresponding densities from Osten et al.'s emission measures would be closer to those of Table 4. An interpretation, already suggested by Osten et al. (2002), can be that the microwave emission may arise mainly in lower density regions further out in the corona than the EUV emission, although, as follows from the definition of $\xi$, it would be possible to increase the ambient plasma density for the radio-emitting locations by making a flatter, lower structure.

The general characteristics of the emission regime, for example that the X-band emission emerges from measurably greater depths than the C-band, could be supported
Table 4. Coronal parameters for gyrosychrotron models

\begin{tabular}{rccc}
\hline$\xi$ & $a=\frac{2}{3}$ & $a=1$ & $a=2$ \\
\hline \multicolumn{3}{c}{$\log B(\mathrm{C}, \mathrm{X})$} \\
-1 & $2.22,2.55$ & $2.06,2.36$ & $1.69,1.54$ \\
0 & $1.99,2.31$ & $1.84,2.15$ & $1.52,1.76$ \\
1 & $1.75,2.08$ & $1.63,1.93$ & $1.34,1.59$ \\
& \multicolumn{3}{c}{$\log L(\mathrm{C}, \mathrm{X})$} \\
-1 & $10.36,10.18$ & $10.30,10.11$ & $10.16,9.95$ \\
0 & $10.28,10.09$ & $10.22,10.02$ & $10.10,9.88$ \\
1 & $10.19,10.00$ & $10.14,9.94$ & $10.03,9.81$ \\
& & $\log N_{\mathrm{e}}(\mathrm{C}, \mathrm{X})$ & \\
-1 & $8.98,9.20$ & $9.56,9.80$ & $10.89,11.38$ \\
0 & $8.83,9.04$ & $9.34,9.65$ & $10.53,11.03$ \\
1 & $8.68,8.89$ & $9.13,9.43$ & $10.18,10.67$ \\
& &
\end{tabular}

The electron densities given in this table are two orders of magnitude larger than those used in equation (2) $\left(\log N_{\mathrm{R}}\right)$, on the basis of $\psi=0.01$ in Equation (3).

by a more detailed treatment of the radiation transfer equation; however, such a procedure calls for more modelling assumptions than can be directly verified by observations. Numerical integration through a model corona would, in the first instance, assume some static or equilibrium density distribution, which the very circumstances of cool star activity tend to contradict. The exercise will, however, show up physical issues that are glossed over in the foregoing simplifications. For example, a coronal structure that becomes optically thick at a certain radius is likely to be above coronal material of greater density and stronger field where the opacity strongly increases, i.e. the situation considered above is difficult to visualise in isolation from an optically thick understructure. This ties in with the field lines associated with coronal emission deriving from those of the maculation regions, some orders of magnitude more intense. The inference is that compact sources of gyrosynchrotron radiation that can account for the quiescent undulations and match the required sizes, fields and temperatures to plausible sources are probably optically thick. Hence, the occasional observations of significant levels of circular polarisation indicate that not all of the observed emission can be gyrosynchrotron emission of the predicted kind.

This leads on to the role of 'microflaring', which has been considered as a contributor to the microwave emission. Our present data show that individual flares can be distinguished, from time to time. What we cannot easily determine is whether more, but less intense, flares could sum together to account for some proportion of the received flux. Qualitatively, the quiescent undulations can be matched by adding together randomly occurring flares that follow an inverse power-law of energy distribution, but other detailed studies of microwave radiation from cool stars (e.g. Slee et al. 1987; Rucinski, Krogulec, \& Seaquist 1993; Jones et al. 1996) have argued against such an interpretation. Observational evidence for an absence of continual weak flaring has 
been obtained at times of strong radio emission, when existing synthesis telescopes would have had sufficient sensitivity at the high time resolution required to recognise weak, short flares. The weak quiescent emissions reported here may, however, contain such structure. This suggestion is supported by the significant correlation between 4.80 and $8.64 \mathrm{GHz}$ intensities found at zero lag in the non-flaring interval on December 30, as reported in Section 2.4.

The strong flare on December 30 (Fig. 4) produced a cross-correlation function (Fig. 5) with a delay of $\sim 5 \mathrm{~min}$ between 4.80 and $8.64 \mathrm{GHz}$ flux densities, which suggests the propagation of a hydromagnetic disturbance out through the corona and exciting gyrosynchrotron emission at increasingly lower frequencies as it travels. Similar effects were reported in a variety of RS CVns and single active stars by Budding et al. (2002) including AB Dor and $\mathrm{AU}$ Mic, which have spectral and luminosity classes similar to those of the components of CC Eri. In this study, we are unable to differentiate between the K7V and M4V components or, indeed, rule out the possibility that the activity identified in the emission of December 30 originated from inter-component magnetic field reconnections.

There is clear evidence from the present observations that neither the quiescent nor the strong flare emissions arise from a coherent process. The quiescent flux density is polarised to the extent of $\sim 20 \%$ (Table 1), but this is not unexpected from gyrosynchrotron emission from a plasma near to optically thin conditions. The brightness temperature derived from equation (1) is high for gyrosynchrotron radiation, but would fall below $10^{9} \mathrm{~K}$ if the source radius were to increase to $\sim 0.3 R_{\star}$. The flares at 4.80 and $8.64 \mathrm{GHz}$ would have considerably higher peak brightness temperatures, especially if attributed to the dwarf component, but their low polarisation fractions, broad bandwidths, and optically thick spectra are evidence against any coherent mechanism.

On the other hand, Osten et al. (2002) derived physical conditions that could not easily be reconciled with emission regimes like those discussed above. Osten et al. remarked that their large average values of $N_{\mathrm{e}}$, deduced from high excitation lines of Fe in CC Eri's EUV spectrum, would not permit microwave emission to escape from the corona. If, however, we adopt their stated lower limit of $N_{\mathrm{e}}=10^{12} \mathrm{~cm}^{-3}$ and postulate a BaumbachAllen type radial variation in the thermal electron density, at $R / R_{\star}=3.5$ we would have $N_{\mathrm{e}}=2.8 \times 10^{9} \mathrm{~cm}^{-3}$, for which $v_{\mathrm{p}}=4.7 \times 10^{8} \mathrm{~Hz}$. We also avoid the RazinTsytovich suppression that would set in at frequencies below about $7.5 \times 10^{8} \mathrm{~Hz}$, the latter limit allowing the emission at $1.4 \mathrm{GHz}$ (detected strongly by Osten et al. 2002) to escape the corona. Sources of the microwave emission could thus be located at such radial distances in the corona [corresponding, perhaps, to around the distance of the 'dead zone' of Mestel and Spruit's (1987, Table 1) coronal models]. However, we would also urge further and more detailed multiwavelength observational studies to give a more comprehensive account of the various likely contributors to the emission from stars like CC Eri.

\section{Conclusions}

We conclude that CC Eri is a flaring BY Dra type binary, whose remarkable magnetic activity is evident at radio wavelengths and also directly observable in the optical range of the spectrum. We can associate its behaviour generally with that of the RS CVn type stars, with similar physical processes underlying the observed phenomena.

We have shown in Section 2.4 that the 'quiescent' emission on December 30 actually consists of a series of low-amplitude intensity fluctuations, which are significantly correlated at 4.80 and $8.64 \mathrm{GHz}$. Cross-correlation of the 4.80 and $8.64 \mathrm{GHz}$ intensities during the strong flare on December 30 yielded a time delay of $\sim 5 \mathrm{~min}$ between the arrival times at the two frequencies with the higher frequency flare preceding that at the lower frequency. We interpret this behaviour as micro- and macro-flaring in the lower corona, resulting in the propagation of hydrodynamic disturbances outwards and accelerating electrons to near relativistic energies as the disturbance progresses. The emission at $8.64 \mathrm{GHz}$ can escape from deeper in the corona than can that at $4.80 \mathrm{GHz}$, thus explaining the observed delay. The lack of strong polarisation and its wide bandwidth preclude a coherent mechanism, while the moderate brightness temperature, comparatively low polarisation, and slightly positive spectral index strongly suggest a gyrosynchrotron source.

In Section 2.5 we demonstrated the presence of a strong surface magnetic field, but since the optical spectropolarimetry was of very limited duration, we were not to able to image the field regions. However, in Sections 2.6 and 2.7, we were able to deduce the likely size and location of a major photospheric maculation region on the brighter component. Keeping in mind that both components of the binary probably possess strong surface fields, we hope to perform more complete Doppler imaging in the polarised emission in a future extended observing session.

In Section 3 an attempt has been made to build a simple model, using the gyrosynchrotron emission formulae developed by Dulk (1985). By assuming that the number of radiating electrons is a given function of the magnetic field in the source region, it is possible to derive feasible values for the field, source radius and ambient electron density. We could also confirm that these values were not strongly dependent on the form of the field modelling function (within reason) or the source's aspect ratio to the observer.

More detailed understanding of these physical mechanisms could be achieved by further detailed multiwavelength studies of this and similar stars, with improved data quality and quantity, if possible. The general aim of such work is to build up a more complete three-dimensional picture of the enormous magnetic structures that must exist on active stars. Physical understanding of cool, solar-type stars can be advanced in this way. 


\section{Acknowledgements}

The Australia Telescope Compact Array is part of the Australia Telescope, which is funded by the Commonwealth of Australia for operation as a National Facility managed by CSIRO.

E.B. gratefully acknowledges the Kingdon-Tomlinson fund, administered by the Royal Astronomical Society of New Zealand in supporting his observational visit to the Australia Telescope National Facility. He also thanks the Director and Staff of the Australia Telescope National Facility for help and hospitality. B.D.C and M.W.M would like to extend sincere thanks to the Director and Staff of the Anglo-Australian Observatory, and to Meir Semel of the Observatoire Paris-Meudon for use of the ZDI SemelPol polarimeter. The AAT observations of B.D.C and M.W.M were supported by a team project grant provided by the University of Southern Queensland. This paper made use of data from the Hipparcos satellite, the Science and Engineering Research Council, SIMBAD, and the NASA Astrophysics Data System.

\section{References}

Allen, C. W. 1974, Astrophysical Quantities (Athlone Press, London) Ayres, T. R., et al. 2000, BAAS, 197, 4407

Bessell, M. S. 1996, in PEP5 Conf. Proc., AustJA, 6, 211

Budding, E. 1977, Ap\&SS, 48, 207

Budding, E. 1993, Introduction to Astronomical Photometry (Cambridge, UK: CUP)

Budding, E., Jones, K. L., Slee, O. B., \& Watson, L. 1999, MNRAS, 305, 966

Budding, E., Lim, J., Slee, O. B., \& White, S. M. 2002, New A, 7, 35

Cameron, A. C., \& Robinson, R. D. 1989, MNRAS, 238, 657

Cutispoto, G., Messina, S., \& Rodono, M. 2001, A\&A, 367, 910

Donati, J.-F., Semel, M., Carter, B. D., Rees, D. E., \& Cameron, A. C. 1997, MNRAS, 291, 658
Dempsey, R. C., Linsky, J. L., Schmitt, J. H. M. M., \& Fleming, T. A. 1993, ApJ, 413, 333

Drake, S. A., Simon, T., \& Linksy, J. L. 1992, ApJS, 82, 311

Dulk, G. A. 1985, ARA\&A, 23, 169

Evans, D. S. 1959, MNRAS, 119, 526

Franciosini, E., \& Chiuderi Drago, F. 1995, A\&A, 297, 535

Gary, D. E. 1985, ApJ, 297, 799

Henry, G. W., Eaton, J.A., Hamer, J., \& Hall, D. S. 1995, ApJS, 97,513

Jardine, M., Cameron, A. C., \& Donati, J.-F. 2002, 333, 339

James, D. J., Jardine, M. M., Jeffries, R. D., Randich, S., Cameron, A. C., \& Ferreira, M. 2000, MNRAS, 318, 1217

Jones, K. L., Brown, A., Stewart, R. T., \& Slee, O. B. 1996, MNRAS, 283,1331

Lane, D. J. 1995, ToFits at ftp://hercules.stmarys.ca/pub/tofits/ to-fits.exe

Manchester, R. 1991, AdSpR, 11, 403

Mestel, L., \& Spruit, H. C. 1987, MNRAS, 226, 57

Osten, R. A., Brown, A., Ayres, T. R., Linsky, J. L., Drake, S. A., Gagné, M., \& Stern, R. A. 2000, ApJ, 544, 953

Osten, R. A., Brown, A., Wood, B. E., \& Brady, P. 2002, ApJS, 138,99

Popper, D. M. 1980, ARA\&A 18, 115.

Rucinski, S. M., Krogulec, M., \& Seaquist, E. R. 1993, AJ, 105, 2308

Sault, R., \& Killeen, N. 1996, Multichannel Image Reconstruction, Image Analysis and Display (MIRIAD), User's Guide, ATNF, CSIRO

Slee, O. B., et al. 1987, MNRAS, 227, 446

Slee, O. B., \& Stewart, R. T. 1989, MNRAS, 236, 129

Stetson, P. B. 1990, PASP 102, 932.

Stewart, R. T., Innis, J. L., Slee, O. B., Nelson, G. J., \& Wright, A. E. 1988, AJ, 96, 371

Strassmeier, K. G., Hall, D. S., Fekel, F. C., \& Scheck, M. 1993, A\&AS, 100, 173

Vilhu, O., Tsuru, T., Cameron, A. C., Budding, E., Banks, T., Slee, B., Ehrenfreund, P., \& Foing, B. H. 1993, A\&A, 278, 467

Waite, I. A. 1999, M. Phil. Thesis, Univ. Southern Queensland, Australia

Watson, L. 1999, PhD Thesis, Univ. Canterbury 